\title{
Superposition in Classes of Ultradifferentiable Functions
}

By

Carmen Fernández* and Antonio Galbis**

\begin{abstract}
We present a complete characterization of the classes of ultradifferentiable functions that are holomorphically closed. Moreover, we show that any class holomorphically closed is also closed under composition (now without restrictions on the number of variables). In this case, we also discuss continuity and differentiability properties of the non-linear superposition operator $g \rightarrow f \circ g$.
\end{abstract}

\section{$\S 0 . \quad$ Introduction}

It is a well-known fact that the composition of two $\mathcal{C}^{\infty}$ (respectively analytic) functions is again $\mathcal{C}^{\infty}$ (resp. analytic). Mainly motivated by the study of (the regularity of) elementary solutions of linear partial differential operators with constant coefficients, several intermediate classes of functions between real analytic and $\mathcal{C}^{\infty}$ functions have been introduced and studied during the last century, and hence it is natural to investigate whether these new classes of functions, known as classes of ultradifferentiable functions, are closed by composition. The first result in this direction seems to be due to M. Gevrey,

Communicated by T. Kawai. Received December 7, 2004

2000 Mathematics Subject Classification(s): 46E25, 46F05, 47H30.

Key words: Superposition, Ultradifferentiable.

The research of the author(s) was partially supported by MEC and FEDER, Project MTM2004-02262, by MCYT and MURST-MIUR Acción Integrada HI 2003-0066 and by AVCIT Grupos 03/050.

* Departamento de Análisis Matemático, Universidad de Valencia, Doctor Moliner 50, 46100 Burjasot (Valencia), Spain e-mail: Carmen.Fdez-Rosell@uv.es

** Departamento de Análisis Matemático, Universidad de Valencia, Doctor Moliner 50, 46100 Burjasot (Valencia), Spain e-mail: Antonio.Galbis@uv.es

(C) 2006 Research Institute for Mathematical Sciences, Kyoto University. All rights reserved. 
who introduced a scale of intermediate spaces, the so-called Gevrey classes, and showed that the composition of two functions in a given class remains in the same class.

A class $\mathcal{F}$ of real or complex valued functions is said to be inverse closed if $1 / f$ remains in the class whenever $f$ is in the class and it does not vanish, and it is said to be holomorphically closed if $F \circ f \in \mathcal{F}$ for every $f \in \mathcal{F}$ and for each function $F$ which is holomorphic on a complex neighborhood of the range of $f$.

The problem of characterizing the Denjoy-Carleman classes which were inverse closed, or equivalently holomorphically closed, was posed by P. Malliavin [19]. In the non-quasianalytic setting and for $2 \pi$-periodic functions the problem was solved by W. Rudin [27], and later extended to general Denjoy-Carleman classes on $\mathbb{R}$ by Boman and Hörmander [6].

Similar results were obtained by Roumieu [26], who studied conditions on a sequence $\left(N_{p}\right)_{p \in \mathbb{N}}$ in order to guarantee that $f \circ g \in \mathcal{E}_{\left\{M_{p}\right\}}$ provided that $f \in \mathcal{E}_{\left\{M_{p}\right\}}, g \in \mathcal{E}_{\left\{N_{p}\right\}}$ and $\mathcal{E}_{\left\{N_{p}\right\}} \subset \mathcal{E}_{\left\{M_{p}\right\}}$ with continuous inclusion. In particular, taking $N_{p}=p$ !, he showed that $f \circ g \in \mathcal{E}_{\left\{M_{p}\right\}}$ if $f \in \mathcal{E}_{\left\{M_{p}\right\}}$ and $g$ is real-analytic. Then, he was able to define $N$-dimensional manifolds of class $\left(N_{p}\right)_{p \in \mathbb{N}}$ and also functions of class $\left(M_{p}\right)_{p \in \mathbb{N}}$ on these manifolds.

More recently Siddiqui and Ider [29] studied the inverse closed spaces of ultradifferentiable function of Roumieu type (with uniform bounds on $\mathbb{R}$ and without requiring logarithmic convexity for the defining sequence), and Bruna [9] considered the same problem for some classes of Beurling type. Using almost analytic extensions, Petzsche and Vogt [25] showed that the classes of ultradifferentiable functions considered by Björck [3] are holomorphically closed. Almost analytic extensions were the main tool used by Dynkin [11] to show that several classes of smooth functions were closed by composition. We also refer to $[1,2]$, where some results concerning the continuity of the non-linear superposition operator are included.

We will present a complete characterization of the classes of ultradifferentiable functions on the real line that are holomorphically closed. Our approach to the classes of ultradifferentiable functions is the one of Braun, Meise and Taylor [8]. In particular, our result applies to the most relevant cases considered by Komatsu [16]. As follows from our results, the behaviour of a given non-quasianalytic class of Beurling type with respect to the problem of being holomorphically closed is similar to that of the corresponding class of Roumieu type. Moreover, we show that any class holomorphically closed is also closed under composition (now without restrictions on the number of variables). In 
this case, we also discuss differentiability properties of the non-linear superposition operator $g \rightarrow f \circ g$.

\section{$\S 1 . \quad$ Preliminaries}

First we introduce the spaces of functions and most of the notation that will be used in the sequel. All definitions are taken from [8].

Definition 1.1. Let $\omega:[0, \infty[\rightarrow[0, \infty[$ be a continuous function which is increasing and satisfies $\omega(0)=0$ and $\omega(1)>0$. Then $\omega$ is called a weight function if it satisfies the following conditions:

$(\alpha) \omega(e t) \leq L(1+\omega(t))$ for some $L \geq 1$ and for all $t>0$.

( $\beta) \int_{1}^{\infty} \frac{\omega(t)}{t^{2}} d t<\infty$.

$(\gamma) \log (t)=o(\omega(t))$ as $t$ tends to $\infty$.

$(\delta) \varphi: t \rightarrow \omega\left(e^{t}\right)$ is convex.

A weight $\omega$ is equivalent to a sub-additive weight if, and only if, $\omega$ has property

$$
\begin{gathered}
\left(\alpha_{0}\right) \quad \exists C_{1} \exists t_{0}>0 \forall \lambda \geq 1 \forall t \geq t_{0}: \\
\omega(\lambda t) \leq \lambda C_{1} \omega(t) .
\end{gathered}
$$

The above condition should be compared with [25, p. 19] and [23, Lemma 1]. The Young conjugate of $\varphi$ is defined by $\varphi^{*}(x)=\sup _{y>0}\{x y-\varphi(y)\}$.

Definition 1.2. Let $\omega$ be a weight function and let $\Omega$ be an open set in $\mathbb{R}^{N}$. We define, $\mathcal{E}_{(\omega)}(\Omega):=\left\{f \in C^{\infty}(\Omega):\|f\|_{K, \lambda}<\infty\right.$, for every $K \subset \subset \Omega$, and every $\left.\lambda>0\right\}$, and

$$
\mathcal{E}_{\{\omega\}}(\Omega):=\left\{f \in C^{\infty}(\Omega) \text { : for every } K \subset \subset \Omega \text {, there exists } \lambda>0\right. \text { such that }
$$

$$
\left.\|f\|_{K, \lambda}<\infty\right\}
$$

where

$$
\|f\|_{K, \lambda}:=\sup _{x \in K} \sup _{\alpha \in \mathbb{N}_{0}^{n}}\left|f^{(\alpha)}(x)\right| \exp \left(-\lambda \varphi^{*}\left(\frac{|\alpha|}{\lambda}\right)\right) .
$$


$\mathcal{E}_{(\omega)}(\Omega)$ is endowed with its natural Fréchet topology, while $\mathcal{E}_{\{\omega\}}(\Omega)$ is a projective limit of $(L B)$ spaces.

The elements of $\mathcal{E}_{(\omega)}(\Omega)$ (resp. $\mathcal{E}_{\{\omega\}}(\Omega)$ ) are called $\omega$-ultradifferentiable functions of Beurling (resp. Roumieu) type. We write $\mathcal{E}_{*}(\Omega)$, where $*$ can be either $(\omega)$ or $\{\omega\}$.We put

$$
\mathcal{D}_{*}(K)=\left\{f \in \mathcal{E}_{*}(\Omega): \operatorname{supp} f \subset K\right\}
$$

and

$$
\mathcal{D}_{*}(\Omega):=\operatorname{ind}_{j \rightarrow} \mathcal{D}_{*}\left(K_{j}\right)
$$

where $\left(K_{j}\right)_{j \in \mathbb{N}}$ denotes a fundamental sequence of compact sets of $\Omega$.

We mention that $\omega(t):=|t|^{1 / d}(d>1)$ are weight functions satisfying property $\left(\alpha_{0}\right)$ and that the corresponding Roumieu class is the Gevrey class with exponent $d$.

From now on, the elements in $\mathcal{E}_{*}(\Omega)$ will be, in general, real valued and we will write $\mathcal{E}_{*}(\Omega ; \mathbb{C})$ for complex valued functions. We will denote by $\mathcal{H}(U)$ the space of holomorphic functions on an open subset $U \subset \mathbb{C}$ and by $\mathcal{A}(\mathbb{R})$ the space of real analytic functions.

\section{§2. The One Variable Case}

The aim of this section is to characterize, in terms of the weight function $\omega$, the classes of ultradifferentiable functions on the real line which are holomorphically closed. For some spaces of ultradifferentiable functions of Beurling type, this was done by Bruna [9]. Petzsche and Vogt [25] showed that this is the case for both the Beurling and the Roumieu case if the weight function is (equivalent to a) sub-additive, using almost analytic extensions.

Our next proposition is an easy application of the Faà di Bruno formula.

Proposition 2.1. $\quad$ Let us assume that $\omega$ satisfies $\left(\alpha_{0}\right)$ and let $f, g \in$ $\mathcal{E}_{*}(\mathbb{R})$ be given. Then $f \circ g \in \mathcal{E}_{*}(\mathbb{R})$. Moreover,

(1) In the case $*=\{\omega\}:$ For every $\lambda>0$ and $C_{1}>0$ there exist $\mu>0$ and $C_{2}>0$ such that the condition $\|g\|_{K, 2 \lambda} \leq C_{1}$ implies $\|f \circ g\|_{K, \mu} \leq C_{2} \|$ $f \|_{g(K), \lambda}$.

(2) In the case $*=(\omega):$ For every $m \in \mathbb{N}$ and $C_{1}>0$ there exist $\ell \in \mathbb{N}$ and $C_{2}>0$ such that the condition $\|g\|_{K, m} \leq C_{1}$ implies $\|f \circ g\|_{K, m} \leq C_{2} \|$ $f \|_{g(K), \ell}$. 
Proof. We fix a compact subset $K \subset \mathbb{R}$ and we take $\lambda>0$ and $C_{1}>0$ such that

$$
\sup _{x \in K}\left|g^{(j)}(x)\right| \leq C_{1} \exp \left(2 \lambda \varphi^{*}\left(\frac{j}{2 \lambda}\right)\right), \quad j=0,1, \ldots .
$$

We apply the Faà di Bruno formula (see e.g. [17, 1.3.1]) to get

$$
(f \circ g)^{(n)}(x)=\sum \frac{n !}{k_{1} ! \ldots k_{n} !} f^{(k)}(g(x))\left(\frac{g^{\prime}(x)}{1 !}\right)^{k_{1}} \ldots\left(\frac{g^{(n)}(x)}{n !}\right)^{k_{n}}
$$

where the sum is extended over all $\left(k_{1}, \ldots, k_{n}\right)$ such that $k_{1}+2 k_{2}+\cdots+n k_{n}=n$ and $k:=k_{1}+\cdots+k_{n}$.

From the convexity of $\varphi^{*}$ one easily gets that

$$
\exp \left(2 \lambda \varphi^{*}\left(\frac{j}{2 \lambda}\right)\right) \leq D_{\lambda} \exp \left(\lambda \varphi^{*}\left(\frac{j-1}{\lambda}\right)\right)
$$

and hence

$$
\left|\frac{g^{(j)}(x)}{j !}\right|^{k_{j}} \leq\left(C_{1} \frac{e^{2 \lambda \varphi^{*}\left(\frac{j}{2 \lambda}\right)}}{j !}\right)^{k_{j}} \leq\left(C_{\lambda} D_{\lambda}\right)^{k_{j}}\left(\frac{e^{\lambda \varphi^{*}\left(\frac{j-1}{\lambda}\right)}}{(j-1) !}\right)^{k_{j}} .
$$

Since $\omega$ satisfies condition $\left(\alpha_{0}\right)$ we can assume, without loss of generality, that $\omega$ is sub-additive $([25,1.1])$. In this case, the sequence $a_{j}:=\frac{1}{j !} \exp \left(\lambda \varphi^{*}\left(\frac{j}{\lambda}\right)\right)$ satisfies

$$
a_{j} a_{k} \leq a_{j+k}
$$

(see [13, Lemma 3.3]). Consequently

$$
\left|\frac{g^{(j)}(x)}{j !}\right|^{k_{j}} \leq\left(C_{1} D_{\lambda}\right)^{k_{j}} \frac{e^{\lambda \varphi^{*}\left(\frac{(j-1) k_{j}}{\lambda}\right)}}{\left((j-1) k_{j}\right) !} .
$$

Since $\sum_{j=1}^{n}(j-1) k_{j}=n-k$ we have, after applying 2.1 once again and taking $B_{\lambda}:=C_{1} D_{\lambda}$

$$
\prod_{j=1}^{n}\left|\frac{g^{(j)}(x)}{j !}\right|^{k_{j}} \leq\left(B_{\lambda}\right)^{k} \frac{e^{\lambda \varphi^{*}\left(\frac{n-k}{\lambda}\right)}}{(n-k) !}
$$

for all $x \in K$.

(a) The Roumieu case $*=\{\omega\}$.

We can assume $\lambda$ small enough so that

$$
C:=\|f\|_{g(K), \lambda}<+\infty \text {. }
$$


Then

$$
\begin{aligned}
\left|(f \circ g)^{(n)}(x)\right| & \leq C \sum \frac{n !}{k_{1} ! \ldots k_{n} !} e^{\lambda \varphi^{*}\left(\frac{k}{\lambda}\right)}\left(B_{\lambda}\right)^{k} \frac{e^{\lambda \varphi^{*}\left(\frac{n-k}{\lambda}\right)}}{(n-k) !} \\
& \leq C\left(B_{\lambda}\right)^{n} \sum \frac{k !}{k_{1} ! \ldots k_{n} !} e^{\lambda \varphi^{*}\left(\frac{n}{\lambda}\right)} \\
& =C B_{\lambda}^{n} e^{\lambda \varphi^{*}\left(\frac{n}{\lambda}\right)} 2^{n-1},
\end{aligned}
$$

using the fact that $\sum \frac{k !}{k_{1} ! \ldots k_{n} !}=2^{n-1}$ and $\varphi^{*}\left(\frac{n-k}{\lambda}\right)+\varphi^{*}\left(\frac{k}{\lambda}\right) \leq \varphi^{*}\left(\frac{n}{\lambda}\right)$. We now consider $s \in \mathbb{N}$ such that $\left(2 B_{\lambda}\right)^{n} \leq e^{n s}$ and we take $L \geq 1$ as in $(\alpha)$. Then, for $\mu:=\lambda L^{-s}$ we obtain $[14,1.1 .18]$

$$
\lambda \varphi^{*}\left(\frac{n}{\lambda}\right)+n s \leq \mu \varphi^{*}\left(\frac{n}{\mu}\right)+\mu \sum_{j=1}^{s} L^{j} .
$$

Hence

$$
\left|(f \circ g)^{(n)}(x)\right| \leq C_{2} C e^{\mu \varphi^{*}\left(\frac{n}{\mu}\right)}
$$

for some $C_{2}>0$ and for all $x \in K, n \in \mathbb{N}$ and we conclude that $f \circ g \in \mathcal{E}_{\{\omega\}}(\mathbb{R})$.

(b) The Beurling case $*=(\omega)$.

We fix $m \in \mathbb{N}$ and we find $\ell \in \mathbb{N}$ and $\tilde{D}_{m}$ such that

$$
\ell \varphi^{*}\left(\frac{k}{\ell}\right)+k \log B_{m} \leq \widetilde{D}_{m}+m \varphi^{*}\left(\frac{k}{m}\right)
$$

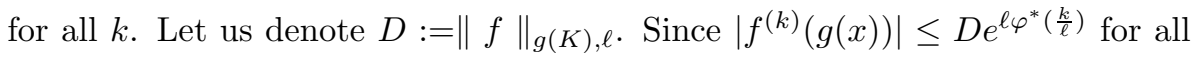
$x \in K, k \in \mathbb{N}_{0}$, we have (taking $\lambda=m$ in the estimates above)

$$
\begin{aligned}
\left|(f \circ g)^{(n)}(x)\right| & \leq \sum \frac{n !}{k_{1} ! \ldots k_{n} !} D e^{\ell \varphi^{*}\left(\frac{k}{\ell}\right)}\left(B_{m}\right)^{k} \frac{e^{m \varphi^{*}\left(\frac{n-k}{m}\right)}}{(n-k) !} \\
& \leq D e^{\widetilde{D}_{m}} \sum \frac{n !}{k_{1} ! \ldots k_{n} !} e^{m \varphi^{*}\left(\frac{k}{m}\right)} \frac{e^{m \varphi^{*}\left(\frac{n-k}{m}\right)}}{(n-k) !} \\
& \leq D C_{2} e^{m \varphi^{*}\left(\frac{n}{m}\right)} 2^{n-1}
\end{aligned}
$$

for all $x \in K, n \in \mathbb{N}_{0}$ (and for some $C_{2}>0$ depending on $m$ ). Hence $f \circ g \in$ $\mathcal{E}_{(\omega)}(\mathbb{R})$.

The use of almost analytic extensions as in [25], gives a different proof of the above Proposition in the Roumieu setting. With the same argument we recover $[25,3.6]$.

Proposition 2.2. For a weight with the property $\left(\alpha_{0}\right)$, the conditions $f \in \mathcal{H}(\mathbb{C})$ and $g \in \mathcal{E}_{*}(\mathbb{R} ; \mathbb{C})$ imply $f \circ g \in \mathcal{E}_{*}(\mathbb{R} ; \mathbb{C})$. 
Now we analyze the necessity of condition $\left(\alpha_{0}\right)$. According to a theorem of Mitiagin, Zelazko and Rolewicz [20] (see also [12]), a Fréchet algebra $A$ (over the field $\mathbb{K}$ of real or complex numbers) is locally m-convex if, and only if, for every $a \in A$ and for every entire function $\phi(z)=\sum_{n=0}^{\infty} c_{n} z^{n}$ (with coefficients $\left.c_{n} \in \mathbb{K}\right)$, the series $\sum_{n=0}^{\infty} c_{n} a^{n}$ converges in $A$. The next argument is taken from [9].

Let us assume that the Fréchet algebra $\mathcal{E}_{(\omega)}(\mathbb{R} ; \mathbb{C})$ is holomorphically closed. Then, by $[20], \mathcal{E}_{(\omega)}(\mathbb{R} ; \mathbb{C})$ is a locally m-convex algebra. Therefore we find a continuous multiplicative seminorm $q$, positive constants $C, B, a$ and $k \in \mathbb{N}$ such that for each $f \in \mathcal{E}_{(\omega)}(\mathbb{R} ; \mathbb{C})$ and each $m \in \mathbb{N}$,

$$
\left\|f^{m}\right\|_{[-1,1], 1} \leq C q\left(f^{m}\right) \leq C(q(f))^{m} \leq C\left(B\|f\|_{[-a, a], k}\right)^{m}
$$

in particular, for $f_{t}(x):=e^{i t x}$ the inequalities above imply that

$$
\exp (\omega(t m)-\log (t m)) \leq C B^{m} \exp (m k \omega(t)) .
$$

It easily follows that $\omega$ satisfies $\left(\alpha_{0}\right)$.

In order to get a similar result for the Roumieu classes we need a different argument since, as shown in [31], there are (non metrizable) commutative algebras in which all entire functions operate but which are not locally multiplicative convex.

We observe that the Beurling class $\mathcal{E}_{(\omega)}(\mathbb{R} ; \mathbb{C})$ is contained in the Roumieu class $\mathcal{E}_{\{\omega\}}(\mathbb{R} ; \mathbb{C})$. Hence, the next proposition implies that the condition $\left(\alpha_{0}\right)$ is necessary in order that the conditions $h \in \mathcal{H}(\mathbb{C})$ and $f \in \mathcal{E}_{*}(\mathbb{R} ; \mathbb{C})$ imply $h \circ f \in \mathcal{E}_{*}(\mathbb{R} ; \mathbb{C}), *$ being $(\omega)$ or $\{\omega\}$.

For a test function $\varphi \in \mathcal{D}_{(\omega)}(\mathbb{R})$ we put

$$
P_{k}(\varphi):=\sup _{t \in \mathbb{R}} \sup _{j \in \mathbb{N}_{0}}\left|\varphi^{(j)}(t)\right| e^{-k \varphi^{*}\left(\frac{j}{k}\right)} .
$$

Proposition 2.3. Let $\omega$ be a weight function and let us assume that, for any $h \in \mathcal{H}(\Omega)$ and $f \in \mathcal{E}_{(\omega)}(\mathbb{R} ; \mathbb{C})$, the condition $f(\mathbb{R}) \subset \Omega$ implies $h \circ f \in$ $\mathcal{E}_{\{\omega\}}(\mathbb{R} ; \mathbb{C})$. Then, $\omega$ satisfies condition $\left(\alpha_{0}\right)$.

Proof. We fix an increasing sequence $\left(t_{j}\right), 0<t_{j}<t_{j+1}<1$, and, for each $j \in \mathbb{N}$ we select $\psi_{j} \in \mathcal{D}_{(\omega)}\left[t_{j}, t_{j+1}\right], 0 \leq \psi_{j} \leq 1$, a test function which is constant equal to 1 on a neighborhood of $b_{j}:=\frac{1}{2}\left(t_{j}+t_{j+1}\right)$.

Let us assume that $\omega$ does not satisfy property $\left(\alpha_{0}\right)$. Then, there are two increasing sequences $\left(k_{n}\right) \subset \mathbb{N}$ and $\left(\xi_{n}\right) \subset \mathbb{R}$ such that

$$
\frac{\omega\left(k_{n} \xi_{n}\right)}{k_{n} \omega\left(\xi_{n}\right)} \geq n^{2}
$$


and $\xi_{n}$ is large enough so that $\sum_{n=1}^{\infty} e^{-n \omega\left(\xi_{n}\right)}<1$ and

$$
\sum_{n=1}^{\infty} e^{-\omega\left(\xi_{n}\right)} P_{n}\left(\psi_{n}\right)<+\infty .
$$

We consider $a_{n}:=e^{-n \omega\left(\xi_{n}\right)}$, define $f_{n}(t):=a_{n} e^{i \xi_{n}\left(t-b_{n}\right)}, n \in \mathbb{N}$, and prove that $f:=\sum_{n=1}^{\infty} f_{n} \psi_{n} \in \mathcal{D}_{(\omega)}[0,1]$. In fact, for any $m \in \mathbb{N}$ there are $C>0$ and $k \in \mathbb{N}$ such that

$$
P_{m}\left(f_{n} \psi_{n}\right) \leq C P_{k}\left(f_{n}\right) P_{k}\left(\psi_{n}\right), n \in \mathbb{N} \text {. }
$$

Since

$$
P_{k}\left(f_{n}\right)=\left|a_{n}\right| \sup _{j \in \mathbb{N}_{0}}\left|\xi_{n}\right|^{j} e^{-k \varphi^{*}\left(\frac{j}{k}\right)} \leq e^{-(n-k) \omega\left(\xi_{n}\right)}
$$

then

$$
\begin{aligned}
\sum_{n=k+1}^{\infty} P_{m}\left(f_{n} \psi_{n}\right) & \leq C \sum_{n=k+1}^{\infty} e^{-(n-k) \omega\left(\xi_{n}\right)} P_{n}\left(\psi_{n}\right) \\
& \leq C \sum_{n=k+1}^{\infty} e^{-\omega\left(\xi_{n}\right)} P_{n}\left(\psi_{n}\right)<+\infty
\end{aligned}
$$

This shows that the series $\sum_{n=1}^{\infty} f_{n} \psi_{n}$ converges to a function $f \in \mathcal{D}_{(\omega)}[0,1]$ and $f(\mathbb{R}) \subset \mathbb{D}$.

By hypothesis,

$$
T_{f}: \mathcal{H}(\mathbb{D}) \rightarrow \mathcal{E}_{\{\omega\}}(\mathbb{R} ; \mathbb{C}), \quad h \rightarrow h \circ f
$$

is a well-defined continuous and linear map (by the closed graph theorem $[15$, 5.4.1]). Since $\mathcal{B}:=\left\{z^{k}\right\}$ is a bounded set in $\mathcal{H}(\mathbb{D})$ then $T_{f}(\mathcal{B})=\left\{f^{k}: k \in \mathbb{N}\right\}$ is a bounded set in $\mathcal{E}_{\{\omega\}}(\mathbb{R} ; \mathbb{C})$. Since $f=f_{n}$ in a neighborhood of $b_{n}$ we have, for some $\mu>0$,

$$
\sup _{n \in \mathbb{N}} \sup _{k \in \mathbb{N}} \sup _{j \in \mathbb{N}}\left|\left(f_{n}^{k}\right)^{(j)}\left(b_{n}\right)\right| e^{-\mu \varphi^{*}\left(\frac{j}{\mu}\right)}<+\infty,
$$

which implies,

$$
\sup _{n \in \mathbb{N}} \sup _{k \in \mathbb{N}} \sup _{j \in \mathbb{N}} a_{n}^{k}\left|\xi_{n} k\right|^{j} e^{-\mu \varphi^{*}\left(\frac{j}{\mu}\right)}<+\infty .
$$

As $\frac{\mu}{2} \omega\left(k_{n} \xi_{n}\right) \leq \sup _{j \in \mathbb{N}}\left(j \log \left|k_{n} \xi_{n}\right|-\mu \varphi^{*}\left(\frac{j}{\mu}\right)\right)$, we deduce

$$
-n k_{n} \omega\left(\xi_{n}\right)+\frac{\mu}{2} \omega\left(k_{n} \xi_{n}\right) \leq C
$$

for some constant $C>0$ and for all $n \in \mathbb{N}$. This contradicts the selection of $\left(k_{n}\right)$ and $\left(\xi_{n}\right)$. 


\section{$\S 3 . \quad$ From one to Several Variables}

In the previous section we have obtained a complete characterization of those non-quasianalytic classes of ultradifferentiable functions which are holomorphically closed in terms of the weight function, and have shown that these classes are closed by composition. Now, we want to extend this result for higher dimensions. One could try to compute the partial derivatives of a composition of two functions. An explicit expression of the partial derivatives of $f \circ g$ for several variables, that is a multivariate Faà di Bruno formula, is given in [10]. However it seems too cumbersome. In this section we provide a onedimensional characterization of the classes of ultradifferentiable functions of $N$ variables, which should be compared with [11, Theorem 1] and [21]. This permits us (in combination with tensor product techniques) to analyze a composition $f \circ\left(g_{1}, \ldots, g_{k}\right)$, where $f \in \mathcal{E}_{*}\left(\mathbb{R}^{k}\right)$ and $g_{1}, \ldots, g_{k} \in \mathcal{E}_{*}\left(\mathbb{R}^{N}\right)$. Let us recall that, as shown independently by Bochnak [4] and Siciak [28], a $\mathcal{C}^{\infty}$ function that is real analytic on every line must be real analytic.

We start with the following result which can be found in [30, p. 226].

Lemma 3.1. Let $P(t)=\sum_{j=0}^{n} a_{j} t^{j}$ be a polynomial of degree less than or equal to $n$. Then,

$$
\left|a_{j}\right| \leq \frac{n^{j}}{j !} \max _{-1 \leq t \leq 1}|P(t)|
$$

An induction argument gives

Lemma 3.2. Let $P(x)=\sum_{|\alpha|=k} a_{\alpha} x^{\alpha}, x \in \mathbb{R}^{N}$, be a homogeneous polynomial of degree $k$. Then

$$
\left|a_{\alpha}\right| \leq e^{k N} \max _{\|x\|_{\infty}=1}|P(x)|
$$

Proof. We proceed by induction on the dimension $N$. For $N=1$ this is obvious. Let us assume that the lemma is true for homogeneous polynomials on $\mathbb{R}^{N-1}, N \geq 2$.

Now we put $x=(y, t) \in \mathbb{R}^{N-1} \times \mathbb{R}, \alpha=(\beta, j)$, and

$$
P(x)=\sum_{j=0}^{k}\left(\sum_{|\beta|=k-j} a_{(\beta, j)} y^{\beta}\right) t^{j} .
$$

We denote $M:=\max _{\|x\|_{\infty}=1}|P(x)|$ and we fix $y \in \mathbb{R}^{N-1}$ with $\|y\|_{\infty}=1$. 
For every $-1 \leq t \leq 1$ we have

$$
\left|\sum_{j=0}^{k}\left(\sum_{|\beta|=k-j} a_{(\beta, j)} y^{\beta}\right) t^{j}\right| \leq M,
$$

and we can apply Lemma 3.1 to get

$$
\max _{\|y\|_{\infty}=1}\left|\sum_{|\beta|=k-j} a_{(\beta, j)} y^{\beta}\right| \leq M e^{k}
$$

and the estimate holds for each $0 \leq j \leq k$. Since $\sum_{|\beta|=k-j} a_{(\beta, j)} y^{\beta}$ is a homogeneous polynomial of degree $k-j$ in $N-1$ variables, we obtain by hypothesis

$$
\left|a_{(\beta, j)}\right| \leq e^{(k-j)(N-1)} M e^{k} \leq M e^{k N}
$$

and the proof is finished.

In the next result $f_{a, v}(t):=f(a+t v), t \in \mathbb{R}$, and $\|v\|_{1}:=\sum_{j=1}^{N}\left|v_{j}\right|$.

Proposition 3.3. Let $f \in \mathcal{D}\left(\mathbb{R}^{N}\right)$ and $\mathcal{B}:=\left\{f_{a, v}: a \in \mathbb{R}^{N},\|v\|_{1}=1\right\}$ be given. Then $f \in \mathcal{D}_{(\omega)}\left(\mathbb{R}^{N}\right)$ (resp. $f \in \mathcal{D}_{\{\omega\}}\left(\mathbb{R}^{N}\right)$ ) if and only if

$$
\sup _{h \in \mathcal{B}}\|h\|_{[-1,1], \lambda}<+\infty
$$

for every $\lambda>0$ (resp. for some $\lambda>0)$.

Proof. Let us assume $f \in \mathcal{D}_{*}\left(\mathbb{R}^{N}\right)$ and $|f|_{\lambda}<\infty$, where

$$
|f|_{\lambda}:=\sup _{x \in \mathbb{R}^{N}} \sup _{\alpha \in \mathbb{N}_{0}^{N}}\left|f^{(\alpha)}(x)\right| e^{-\lambda \varphi^{*}\left(\frac{|\alpha|}{\lambda}\right)} .
$$

We fix $a \in \mathbb{R}^{N},\|v\|_{1}=1$ and we take $\varphi:=f_{a, v}$. Then

$$
\varphi^{(k)}(t)=\sum_{i_{1}, \ldots, i_{k}=1}^{N} v_{i_{1}} \cdots v_{i_{k}} D_{i_{1} \ldots i_{k}} f(a+t v) .
$$

Hence

$$
\left|\varphi^{(k)}(t)\right| \leq|f|_{\lambda} \sum_{i_{1}, \ldots, i_{k}=1}^{N}\left|v_{i_{1}} \cdots v_{i_{k}}\right| e^{\lambda \varphi^{*}\left(\frac{k}{\lambda}\right)}=|f|_{\lambda} e^{\lambda \varphi^{*}\left(\frac{k}{\lambda}\right)}\|v\|_{1}^{k}=|f|_{\lambda} e^{\lambda \varphi^{*}\left(\frac{k}{\lambda}\right)},
$$


and

$$
\sup _{|t| \leq 1} \sup _{k \in \mathbb{N}_{0}}\left|\varphi^{(k)}(t)\right| e^{-\lambda \varphi^{*}\left(\frac{k}{\lambda}\right)} \leq|f|_{\lambda}
$$

for every $a \in \mathbb{R}^{N}$ and $v \in \mathbb{R}^{N}$ with $\|v\|_{1}=1$.

Conversely, let us assume that $\mathcal{B}$ satisfies the condition (3.2). Given $v=$ $\left(v_{1}, \ldots, v_{n}\right)$ and $\alpha=\left(\alpha_{1}, \ldots, \alpha_{n}\right)$ we put $v^{\alpha}:=v_{1}^{\alpha_{1}} \cdots v_{n}^{\alpha_{n}}$. By assumption, in the Beurling case, for each $\lambda$ there is $M>0$ (there are $\lambda, M$ in the Roumieu case) such that

$$
\sup _{-1 \leq t \leq 1}\left|\sum_{i_{1}, \ldots, i_{k}=1}^{N} v_{i_{1}} \cdots v_{i_{k}} D_{i_{1} \ldots i_{k}} f(a+t v)\right| e^{-\lambda \varphi^{*}\left(\frac{k}{\lambda}\right)} \leq M
$$

for all $a \in \mathbb{R}^{N}$ and $v \in \mathbb{R}^{N}$ with $\|v\|_{1}=1$. This means

$$
\sup _{-1 \leq t \leq 1}\left|\sum_{|\alpha|=k} v^{\alpha} \frac{k !}{\alpha !} f^{(\alpha)}(a+t v)\right| \leq M e^{\lambda \varphi^{*}\left(\frac{k}{\lambda}\right)}
$$

whenever $a \in \mathbb{R}^{N}$ and $v \in \mathbb{R}^{N}$ with $\|v\|_{1}=1$ in particular, taking $t=0$,

$$
\sup _{\|v\|_{1}=1}\left|\sum_{|\alpha|=k} v^{\alpha} \frac{k !}{\alpha !} f^{(\alpha)}(a)\right| \leq M e^{\lambda \varphi^{*}\left(\frac{k}{\lambda}\right)} .
$$

Since $P(v):=\sum_{|\alpha|=k} v^{\alpha} \frac{k !}{\alpha !} f^{(\alpha)}(a)$ is a homogeneous polynomial of degree $k$ in $\mathbb{R}^{N}$, an application of the Lemma 3.2 yields

$$
\left|f^{(\alpha)}(a)\right| \leq e^{k N} N^{k} M e^{\lambda \varphi^{*}\left(\frac{k}{\lambda}\right)}
$$

for $a \in \mathbb{R}^{N}$ and $|\alpha|=k$. We put $\lambda=\mu L^{2 N}$ for the constant $L \geq 1$ as in $(\alpha)$. As in the proof of 2.1 , it follows that $[14,1.1 .18]$

$$
\sup _{x \in \mathbb{R}^{N}} \sup _{\alpha \in \mathbb{N}_{0}^{N}}\left|f^{(\alpha)}(x)\right| e^{-\mu \varphi^{*}\left(\frac{|\alpha|}{\mu}\right)}<\infty .
$$

Corollary 3.4. Let us assume that $\omega$ satisfies $\left(\alpha_{0}\right)$ and let be given real-valued functions $f \in \mathcal{E}_{*}(\mathbb{R})$ and $g \in \mathcal{E}_{*}\left(\mathbb{R}^{N}\right)$. Then $f \circ g \in \mathcal{E}_{*}\left(\mathbb{R}^{N}\right)$.

Proof. We fix $\chi \in \mathcal{D}_{*}\left(\mathbb{R}^{N}\right)$ and we consider $f \circ(\chi g)-f(0) \in \mathcal{D}\left(\mathbb{R}^{N}\right)$.

The proposition above implies that $\left\{(\chi g)_{a, v}: a \in \mathbb{R}^{N},\|v\|_{1}=1\right\}$ is a bounded set in $\mathcal{E}_{*}(\mathbb{R})$ and then, the proof of Proposition 2.1 gives that $\{(f \circ$ 
$\left.\chi g)_{a, v}: a \in \mathbb{R}^{N},\|v\|_{1}=1\right\}$ is a bounded set in $\mathcal{E}_{*}(\mathbb{R})$. Applying again the previous proposition we conclude that $f \circ(\chi g)-f(0) \in \mathcal{D}_{*}\left(\mathbb{R}^{N}\right)$. Since $\chi$ is arbitrary we deduce that $f \circ g \in \mathcal{E}_{*}\left(\mathbb{R}^{N}\right)$.

Proposition 3.5. Let $\omega$ be a weight function satisfying $\left(\alpha_{0}\right)$, let $f \in$ $\mathcal{E}_{*}\left(\mathbb{R}^{k}\right)$ and real valued functions $g_{1}, \ldots, g_{k} \in \mathcal{E}_{*}\left(\mathbb{R}^{N}\right)$ be given. Then $f \circ$ $\left(g_{1}, \ldots, g_{k}\right) \in \mathcal{E}_{*}\left(\mathbb{R}^{N}\right)$.

Proof. For every $1 \leq j \leq k$ we consider the linear and continuous operator

$$
C_{j}: \mathcal{E}_{*}(\mathbb{R}) \rightarrow \mathcal{E}_{*}\left(\mathbb{R}^{N}\right), \varphi \rightarrow \varphi \circ g_{j} .
$$

Let $B$ denote the $k$-linear and continuous map

$$
B: \mathcal{E}_{*}\left(\mathbb{R}^{N}\right) \times \cdots \times \mathcal{E}_{*}\left(\mathbb{R}^{N}\right) \longrightarrow \mathcal{E}_{*}\left(\mathbb{R}^{N}\right), B\left(\psi_{1}, \ldots, \psi_{k}\right)=\psi_{1} \cdots \psi_{k},
$$

and $\Delta: \mathcal{E}_{*}\left(\mathbb{R}^{N}\right) \otimes_{\pi} \cdots \otimes_{\pi} \mathcal{E}_{*}\left(\mathbb{R}^{N}\right) \rightarrow \mathcal{E}_{*}\left(\mathbb{R}^{N}\right)$ the induced map. Then, $S:=$ $\Delta \circ\left(C_{1} \otimes \cdots \otimes C_{k}\right)$ is a continuous and linear map

$$
S: \mathcal{E}_{*}(\mathbb{R}) \otimes_{\pi} \cdots \otimes_{\pi} \mathcal{E}_{*}(\mathbb{R}) \longrightarrow \mathcal{E}_{*}\left(\mathbb{R}^{N}\right) .
$$

Moreover, for $f=\varphi_{1} \otimes \cdots \otimes \varphi_{k}$ we have

$$
\begin{aligned}
S(f)(x) & =\Delta\left(C_{1} \varphi_{1} \otimes \cdots \otimes C_{k} \varphi_{k}\right)(x) \\
& =\varphi_{1}\left(g_{1}(x)\right) \cdots \varphi_{k}\left(g_{k}(x)\right) \\
& =f\left(g_{1}(x), \ldots, g_{k}(x)\right) .
\end{aligned}
$$

Since $\mathcal{E}_{*}(\mathbb{R}) \otimes_{\pi} \ldots{ }^{k)} \otimes_{\pi} \mathcal{E}_{*}(\mathbb{R})$ is a topological vector dense subspace of $\mathcal{E}_{*}\left(\mathbb{R}^{k}\right)$, [8, 8.1] we may extend $S$ as a continuous and linear map $\tilde{S}: \mathcal{E}_{*}\left(\mathbb{R}^{k}\right) \rightarrow \mathcal{E}_{*}\left(\mathbb{R}^{N}\right)$, and since each $f \in \mathcal{E}_{*}\left(\mathbb{R}^{k}\right)$ can be approximated by elements in $\mathcal{E}_{*}(\mathbb{R}) \otimes_{\pi} \ldots{ }^{k)} \otimes_{\pi}$ $\mathcal{E}_{*}(\mathbb{R})$, we have that $\tilde{S}(f)=f \circ\left(g_{1}, \ldots, g_{k}\right)$. In particular $f \circ\left(g_{1}, \ldots, g_{k}\right) \in$ $\mathcal{E}_{*}\left(\mathbb{R}^{N}\right)$, as desired.

Corollary 3.6. Let $\omega$ be a weight function satisfying $\left(\alpha_{0}\right)$. Let $\Omega \subset$ $\mathbb{R}^{N}$ be open. Let $g_{1}, \ldots, g_{k} \in \mathcal{E}_{*}(\Omega)$ be real valued functions such that $g=$ $\left(g_{1}, \ldots, g_{k}\right)$ satisfies $g(\Omega) \subset U$ and $U \subset \mathbb{R}^{k}$ is open. Then $f \circ\left(g_{1}, \ldots, g_{k}\right) \in$ $\mathcal{E}_{*}(\Omega)$ for each $f \in \mathcal{E}_{*}(U)$.

Proof. Fix $x_{0} \in \Omega$ and take $\psi \in \mathcal{D}_{*}(\Omega)$ identically 1 on a neighborhood of $x_{0}$. Let $\chi \in \mathcal{D}_{*}(U)$ be identically 1 on a neighborhood of $g\left(x_{0}\right)$. As we have seen, $h=(\chi) \circ(\psi g) \in \mathcal{E}_{*}\left(\mathbb{R}^{N}\right)$. Since $h$ and $f \circ g$ coincide on a neighborhood of $x_{0}$, the conclusion follows. 
Corollary 3.7. Let $\omega$ and $\sigma$ be two weights such that $\int_{1}^{\infty} \frac{\omega(s t)}{t^{2}} d t=$ $O(\sigma(s))$ as $s \rightarrow \infty$. If $f \in \mathcal{E}_{(\sigma)}\left(\mathbb{R}^{k}\right)\left(\right.$ resp. $\left.\mathcal{E}_{\{\sigma\}}\left(\mathbb{R}^{k}\right)\right)$ and $g_{1}, \ldots, g_{k} \in \mathcal{E}_{(\sigma)}\left(\mathbb{R}^{N}\right)$ $\left(\right.$ resp. $\left.\mathcal{E}_{\{\sigma\}}\left(\mathbb{R}^{N}\right)\right)$ then $f \circ\left(g_{1}, \ldots, g_{k}\right) \in \mathcal{E}_{(\omega)}\left(\mathbb{R}^{N}\right)\left(\right.$ resp. $\left.\mathcal{E}_{\{\omega\}}\left(\mathbb{R}^{N}\right)\right)$.

Proof. We put

$$
\tau(s):=\int_{1}^{\infty} \frac{\omega(s t)}{t^{2}} d t=s \int_{s}^{\infty} \frac{\omega(t)}{t^{2}} d t .
$$

Then, $\tau$ is a sub-additive weight function and $\omega \leq \tau=O(\sigma)$. The conclusion follows.

Summarizing all the previous results we obtain

Theorem 3.8. Let $\omega$ be a weight function. The following conditions are equivalent:

(1) $\omega$ satisfies condition $\left(\alpha_{0}\right)$.

(2) For each $g=\left(g_{1}, \ldots, g_{k}\right): \Omega \subset \mathbb{R}^{N} \rightarrow \mathbb{R}^{k}$ such that $g_{j} \in \mathcal{E}_{*}(\Omega)$ and $g(\Omega) \subset U \subset \mathbb{R}^{k}$ and for each $f \in \mathcal{E}_{*}(U)$, one has $f \circ g \in \mathcal{E}_{*}(\Omega)$.

(3) For every $g \in \mathcal{E}_{*}(\mathbb{R})$ and $f \in \mathcal{H}(\Omega), \Omega \subset \mathbb{C}$ open, the condition $g(\mathbb{R}) \subset \Omega$ implies $f \circ g \in \mathcal{E}_{*}(\mathbb{R})$.

\section{$\S 4$. The Non-linear Superposition Operator}

In this section we will show that whenever composition is defined (in the frame of ultradifferentiable functions) the non-linear superposition operator

$$
\mathcal{E}_{*} \rightarrow \mathcal{E}_{*}, g \rightarrow f \circ g
$$

is continuous. Some differentiability properties are also studied. From now on we will assume that $\omega$ satisfies $\left(\alpha_{0}\right)$.

The next Lemma follows easily from the estimates in the previous sections. Here $\left(K_{n}\right)$ denotes a fundamental sequence of compact sets in $\mathbb{R}^{N}, p_{n}:=n$ $\|\cdot\|_{K_{n}, n}$, which is a fundamental sequence of seminorms in $\mathcal{E}_{(\omega)}\left(\mathbb{R}^{N}\right)$ and $\left(q_{n}\right)$ is a fixed fundamental sequence of seminorms in $\mathcal{E}_{(\omega)}(\mathbb{R})$.

Lemma 4.1. For all $k$ there is $m$ such that for each $C_{1}$ there exists $\ell$ so that if $f \in \mathcal{E}_{(\omega)}(\mathbb{R})$ and $g \in \mathcal{E}_{(\omega)}\left(\mathbb{R}^{N}\right)$ satisfies $p_{m}(g) \leq C_{1}$, then $p_{k}(f \circ g) \leq$ $q_{\ell}(f)$. 
Proposition 4.2. The map

$$
\begin{aligned}
\mathcal{E}_{(\omega)}\left(\mathbb{R}^{k}\right) \times\left(\mathcal{E}_{(\omega)}\left(\mathbb{R}^{N}\right)\right)^{k} & \longrightarrow \mathcal{E}_{(\omega)}\left(\mathbb{R}^{N}\right) \\
\left(f, g_{1}, \ldots, g_{k}\right) & \rightarrow f \circ\left(g_{1}, \ldots, g_{k}\right)
\end{aligned}
$$

is continuous.

Proof. Without loss of generality we assume that $f$ is real valued. Fix a compact convex subset $\mathcal{K}$ in $\mathcal{E}_{(\omega)}\left(\mathbb{R}^{N}\right)$. By the continuity of the product in $\mathcal{E}_{(\omega)}\left(\mathbb{R}^{N}\right)$, given $L \in \mathbb{N}$ we find $r$ such that

$$
p_{L}\left(h_{1} \cdots h_{k}\right) \leq p_{r}\left(h_{1}\right) \cdots p_{r}\left(h_{k}\right) .
$$

For this $r$ we take $m$ as in Lemma 4.1 and $C_{1}:=\max _{h \in \mathcal{K}} p_{m}(h)$. Applying again the Lemma 4.1 we find $\ell$ with

$$
p_{r}(f \circ h) \leq p_{\ell}(f), \forall h \in \mathcal{K} .
$$

Let $g:=\left(g_{1}, \ldots, g_{k}\right) \in \mathcal{K}^{k}$ and $f_{1}, \ldots f_{k} \in \mathcal{E}_{(\omega)}(\mathbb{R})$ be given and put $f=$ $f_{1} \otimes \cdots \otimes f_{k} \in \mathcal{E}_{(\omega)}\left(\mathbb{R}^{k}\right)$. Then $f \circ g=\left(f_{1} \circ g_{1}\right) \cdots\left(f_{k} \circ g_{k}\right)$ hence

$$
p_{L}(f \circ g) \leq p_{r}\left(f_{1} \circ g_{1}\right) \cdots p_{r}\left(f_{k} \circ g_{k}\right) \leq q_{\ell}\left(f_{1}\right) \cdots q_{\ell}\left(f_{k}\right) .
$$

Define $C_{g}: \mathcal{E}_{(\omega)}(\mathbb{R}) \otimes^{k)} \cdots \mathcal{E}_{(\omega)}(\mathbb{R}) \longrightarrow \mathcal{E}_{(\omega)}\left(\mathbb{R}^{N}\right)$ by $C_{g}(f)=f \circ g . C_{g}$ is a linear map and by the estimates above, it is continuous. In fact, the family $\left\{C_{g}: g \in \mathcal{K}^{k}\right\}$ is equicontinuous. Since $\mathcal{E}_{(\omega)}(\mathbb{R}) \otimes^{k)} \cdots \mathcal{E}_{(\omega)}(\mathbb{R})$ is a dense subspace of $\mathcal{E}_{(\omega)}\left(\mathbb{R}^{k}\right)$, we conclude that $\left\{C_{g}: g \in \mathcal{K}^{k}\right\}$ is also equicontinuous as a family of operators from $\mathcal{E}_{(\omega)}\left(\mathbb{R}^{k}\right)$ to $\mathcal{E}_{(\omega)}\left(\mathbb{R}^{N}\right)$, that is, if $\left(r_{\ell}\right)_{\ell}$ is a fundamental sequence of seminorms in $\mathcal{E}_{(\omega)}\left(\mathbb{R}^{k}\right)$ for each $m$ there is $\ell$ so that

$$
p_{m}(f \circ g) \leq r_{\ell}(f)
$$

for each $g \in \mathcal{K}^{k}$.

We take $g:=\left(g_{1}, \ldots, g_{k}\right), h:=\left(h_{1}, \ldots, h_{k}\right) \in \mathcal{K}^{k}$, then

$$
f(g(x))-f(h(x))=\sum_{j=1}^{k}\left(g_{j}(x)-h_{j}(x)\right) \cdot \int_{0}^{1}\left(D_{j} f \circ \alpha_{t}\right)(x) d t
$$

where $\alpha_{t}(x)=h(x)+t(g(x)-h(x)) \in \mathcal{K}^{k}$ for each $0 \leq t \leq 1$. We easily deduce that for each $L$ there is $m$ :

$$
\begin{aligned}
p_{L}(f \circ g-f \circ h) & \leq \sum_{j=1}^{k} p_{m}\left(g_{j}(x)-h_{j}(x)\right) \cdot \int_{0}^{1} p_{m}\left(D_{j} f \circ \alpha_{t}\right) d t \\
& \leq \sum_{j=1}^{k} r_{\ell}\left(D_{j} f\right) p_{m}\left(g_{j}-h_{j}\right) .
\end{aligned}
$$


Therefore, for a fix compact $\mathcal{L}$ in $\mathcal{E}_{(\omega)}\left(\mathbb{R}^{k}\right)$ there is $M$ so that $f_{1}, f_{2} \in \mathcal{L}$ and $g, h \in \mathcal{K}^{k}$ implies

$$
\begin{aligned}
p_{L}\left(f_{1} \circ g-f_{2} \circ h\right) & \leq p_{L}\left(f_{1} \circ g-f_{1} \circ h\right)+p_{L}\left(\left(f_{1}-f_{2}\right) \circ h\right) \\
& \leq M \sum_{j=1}^{k} p_{m}\left(g_{j}-h_{j}\right)+r_{\ell}\left(f_{1}-f_{2}\right) .
\end{aligned}
$$

The proof is complete since $\mathcal{E}_{(\omega)}\left(\mathbb{R}^{k}\right) \times\left(\mathcal{E}_{(\omega)}\left(\mathbb{R}^{N}\right)\right)^{k}$ is metrizable.

Next we analyze the Roumieu case.

Lemma 4.3. Let $\mathcal{B}$ be a bounded set in $\mathcal{E}_{\{\omega\}}\left(\mathbb{R}^{N}\right)$. For each continuous seminorm $p$ in $\mathcal{E}_{\{\omega\}}\left(\mathbb{R}^{N}\right)$ there is a continuous seminorm $q$ in $\mathcal{E}_{\{\omega\}}(\mathbb{R})$ such that $p(f \circ g) \leq q(f)$ for every $f \in \mathcal{E}_{\{\omega\}}(\mathbb{R})$ and each $g \in \mathcal{B}$.

Proof. For a fix $L \subset \subset \mathbb{R}^{N}$ define $\tilde{L}:=\left\{a+v: a \in L,\|v\|_{1}=1\right\}$. Since $\mathcal{B}$ is bounded in $\mathcal{E}_{\{\omega\}}\left(\mathbb{R}^{N}\right)$ there is a compact set $K$ in $\mathbb{R}$ such that $\bigcup\{g(\tilde{L}): g \in \mathcal{B}\} \subset K$. The set $\mathcal{C}:=\left\{g_{a, v}: g \in \mathcal{B}, a \in L,\|v\|_{1}=1\right\}$ is a bounded set in $\mathcal{E}_{\{\omega\}}(\mathbb{R})$. We define $C_{1}(\lambda):=\sup \left\{\|h\|_{[-1,1], 2 \lambda}: h \in \mathcal{C}\right\}$ which is finite if $\lambda$ is small enough $\left(0<\lambda \leq \lambda_{0}\right)$. Using Proposition 2.1 we find $C_{2}(\lambda)$ and $\mu(\lambda)$ such that

$$
\|f \circ h\|_{[-1,1], \mu(\lambda)} \leq C_{2}(\lambda)\|f\|_{h([-1,1]), \lambda} \leq C_{2}(\lambda)\|f\|_{K, \lambda}
$$

for all $h \in \mathcal{C}$; that is, for every $g \in \mathcal{B}$, every $a \in L$ and $\|v\|=1$ we have

$$
\left\|(f \circ g)_{a, v}\right\|_{[-1,1], \mu(\lambda)} \leq C_{2}(\lambda)\|f\|_{K, \lambda} .
$$

Therefore, it follows from (the proof of) Proposition 2.3 that there are $r(\lambda)$ and $C_{3}(\lambda)$ satisfying

$$
\|(f \circ g)\|_{L, r(\lambda)} \leq C_{3}(\lambda) \sup _{a \in L,\|v\|=1}\left\|(f \circ g)_{a, v}\right\|_{[-1,1], \mu(\lambda)} \leq C(\lambda)\|f\|_{K, \lambda},
$$

$\left(0<\lambda \leq \lambda_{0}\right)$ where $C(\lambda)=C_{1}(\lambda) C_{2}(\lambda)$, and the inequality holds for arbitrary $f \in \mathcal{E}_{\{\omega\}}(\mathbb{R})$ and $g \in \mathcal{B}$. Moreover the map $\lambda \rightarrow r(\lambda)$ is an increasing bijection from $] 0, \infty[$ onto itself.

Now, given a continuous seminorm in $\mathcal{E}_{\{\omega\}}\left(\mathbb{R}^{N}\right)$ there exists a compact set $L$ in $\mathbb{R}^{N}$ such that $p$ is a continuous seminorm in $\mathcal{E}_{\{\omega\}}(L)$ and consequently, $p \leq \inf _{n} M_{n}\|\|_{L, r(1 / n)}$ for some sequence $\left(M_{n}\right)_{n}$. It suffices to take

$$
q:=\inf _{n} M_{n} C(1 / n)\|\|_{K, 1 / n}
$$

to conclude. 
Theorem 4.4. For each $f \in \mathcal{E}_{\{\omega\}}\left(\mathbb{R}^{k}\right)$ the non-linear superposition operator

$$
T_{f}:\left(\mathcal{E}_{\{\omega\}}\left(\mathbb{R}^{N}\right)\right)^{k} \longrightarrow \mathcal{E}_{\{\omega\}}\left(\mathbb{R}^{N}\right),\left(g_{1}, \ldots, g_{k}\right) \rightarrow f \circ\left(g_{1}, \ldots, g_{k}\right)
$$

is continuous.

Proof. Using Lemma 4.3 we may show as in the proof of Proposition 4.2 that $T_{f}$ maps bounded sets into bounded sets. On the other hand, it is easy to see that $T_{f}$ is continuous if and only if $T_{f}:\left(\mathcal{D}_{\{\omega\}}\left(\mathbb{R}^{N}\right)\right)^{k} \longrightarrow \mathcal{E}_{\{\omega\}}\left(\mathbb{R}^{N}\right)$ is continuous. Since $\mathcal{D}_{\{\omega\}}\left(\mathbb{R}^{N}\right)$ is an $L N$ - space with compact linking maps (also called Silva space), by $[24,8.5 .28]$ it is enough to see that $T_{f}$ restricted to $\left(\mathcal{D}_{\{\omega\}}\left(\mathbb{R}^{N}\right)\right)^{k}$ is sequentially continuous. This follows from the fact that bounded sets are compact and that the non-linear operator is continuous in the $\mathcal{C}^{\infty}$-setting.

Once we have seen that the composition operator is continuous whenever it is well defined, we would like to study differentiability properties of the operator. Unfortunately, it seems that a satisfactory differential calculus stops at the level of Banach spaces. For instance, as it is stated in [18] "if one looks for infinitely often differentiable mappings, then one ends up with 6 inequivalent notions." We will consider smooth mappings, that is

Definition 4.5 ([18]). Let $E$ be a locally convex space. A curve $c$ : $\mathbb{R} \rightarrow E$ is called differentiable if the derivative $c^{\prime}(t):=\lim _{s \rightarrow 0} \frac{1}{s}(c(t+s)-c(t))$ at $t$ exists for all $t$. A curve $c: \mathbb{R} \rightarrow E$ is called smooth if all the iterated derivatives exist. If $F$ is another locally convex space, a map $f: E \rightarrow F$ is called smooth if it maps smooth curves in $E$ to smooth curves in $F$.

As Boman [5] showed, the smooth mappings on $\mathbb{R}^{N}$ in the previous sense are exactly the usual smooth mappings.

Proposition 4.6. Let $f \in \mathcal{E}_{*}(\mathbb{R})$ be given. The map

$$
T_{f}: \mathcal{E}_{*}\left(\mathbb{R}^{N}\right) \rightarrow \mathcal{E}_{*}\left(\mathbb{R}^{N}\right), g \rightarrow f \circ g
$$

is smooth.

Proof. We put $E:=\mathcal{E}_{*}\left(\mathbb{R}^{N}\right)$ and we fix $\alpha \in C^{\infty}(\mathbb{R}, E)$. We will proceed by induction on $n$ to show that $T_{f} \circ \alpha \in C^{n}(\mathbb{R}, E)$ and

$$
\left(T_{f} \circ \alpha\right)^{(n)}(t)=\sum \frac{n !}{k_{1} ! \ldots k_{n} !}\left(f^{(k)} \circ \alpha(t)\right)\left(\frac{\alpha^{\prime}(t)}{1 !}\right)^{k_{1}} \ldots\left(\frac{\alpha^{(n)}(t)}{n !}\right)^{k_{n}} .
$$


Let us assume $n=1$ and let $\beta(t)=f \circ \alpha(t)$. Given $s, t \in \mathbb{R}(s \neq t)$ we note that, Propositions 4.2 and $4.4, \xi \rightarrow f^{\prime} \circ(\xi \alpha(t)+(1-\xi) \alpha(s))$ is a continuous function from $[0,1]$ to $E$. Consider the vector valued integral

$$
u_{s}:=\int_{0}^{1} f^{\prime} \circ(\xi \alpha(t)+(1-\xi) \alpha(s)) d \xi \in E .
$$

Since $\beta(t)(x)-\beta(s)(x)=f(\alpha(t)(x))-f(\alpha(s)(x))=(\alpha(t)(x)-\alpha(s)(x)) \cdot u_{s}(x)$ then

$$
\frac{\beta(t)-\beta(s)}{t-s}=\frac{\alpha(t)-\alpha(s)}{t-s} \cdot u_{s} .
$$

Since for each continuous seminorm $p$ in $E$,

$$
p\left(u_{s}-f^{\prime} \circ \alpha(t)\right) \leq \int_{0}^{1} p\left(f^{\prime} \circ(\xi \alpha(t)+(1-\xi) \alpha(s))-f^{\prime} \alpha(t)\right) d \xi,
$$

goes to zero as $s \rightarrow t$, we conclude

$$
\lim _{s \rightarrow t} \frac{\beta(t)-\beta(s)}{t-s}=\left(f^{\prime} \circ \alpha(t)\right) \alpha^{\prime}(t),
$$

with convergence in the topology of $E$. Hence the statement is proved for $n=1$. Let us now assume that 4.3 holds for derivatives of order less than or equal to $n$. After replacing $f$ by $f^{(k)}$, we have already proved that the function $\mathbb{R} \rightarrow E$, $t \rightarrow f^{(k)} \circ \alpha(t)$ has derivative $\left(f^{(k+1)} \circ \alpha(t)\right) \alpha^{\prime}(t)$. It follows from 4.3 that $\Phi: t \rightarrow\left(T_{f} \circ \alpha\right)^{(n)}(t)$ is derivable. In order to evaluate the derivative we fix $x \in \mathbb{R}^{N}$ and we put $\gamma: \mathbb{R} \rightarrow \mathbb{R}, \gamma(t):=\left\langle\delta_{x}, \alpha(t)\right\rangle=\alpha(t)(x)$. We now observe that

$$
\begin{aligned}
\left\langle\delta_{x}, \Phi(t)\right\rangle & =\sum \frac{n !}{k_{1} ! \ldots k_{n} !} f^{(k)}(\gamma(t))\left(\frac{\gamma^{\prime}(t)}{1 !}\right)^{k_{1}} \ldots\left(\frac{\gamma^{(n)}(t)}{n !}\right)^{k_{n}} \\
& =(f \circ \gamma)^{(n)}(t) .
\end{aligned}
$$

Finally, an application of Fàa di Bruno formula gives 4.3 for $n+1$.

If the composition operator is defined by a real analytic function we may expect a better behavior.

Definition $4.7([18])$. Let $E$ be a locally convex space. A curve $\alpha$ : $\mathbb{R} \rightarrow E$ is called weakly real analytic or simply real analytic if $u \circ \alpha$ is a real analytic function for every $u \in E^{\prime}$, and we write $\alpha \in \mathcal{A}(\mathbb{R}, E)$.

A curve $\alpha: \mathbb{R} \rightarrow E$ is called topologically real analytic, and we write $\alpha \in \mathcal{A}_{t}(\mathbb{R}, E)$, if for every $t \in \mathbb{R}$ there are $\varepsilon>0$ and $a_{j} \in E$ such that $\alpha(s)=\sum_{j=0}^{\infty} a_{j}(s-t)^{j}$ for $|s-t|<\varepsilon$ and the series converges in $E$. 
If $F$ is another locally convex space, a map $f: E \rightarrow F$ is called (topologically) real analytic if it maps (topologically) real analytic curves in $E$ to (topologically) real analytic curves in $F$.

In the next lemma we do not assume that the class $\mathcal{E}_{*}(\mathbb{R})$ is closed by composition.

Lemma 4.8. Let $f \in \mathcal{A}(\mathbb{R})$ (resp. $f \in \mathcal{H}(\mathbb{C})$ ) be given and we consider $\phi: \mathbb{R} \rightarrow \mathcal{E}_{*}(\mathbb{R})$ given by $\phi(t):=f(\cdot+t)$. Then $\phi \in \mathcal{A}\left(\mathbb{R}, \mathcal{E}_{*}(\mathbb{R})\right)($ resp. $\phi \in$ $\left.\mathcal{A}_{t}\left(\mathbb{R}, \mathcal{E}_{*}(\mathbb{R})\right)\right)$.

Proof. Fix $\mu \in \mathcal{E}_{*}^{\prime}(\mathbb{R})$ and denote $K:=\operatorname{supp} \mu$. For some $\epsilon>0$ the function $f$ admits an holomorphic extension to the open set $\Omega_{\epsilon}:=\{z \in$ $\mathbb{C} \mid \mathrm{d}(z, K)<\epsilon\}$. We fix $0<\delta<\frac{\epsilon}{2}$. Then, for every $|z|<\delta, f(\cdot+z) \in \mathcal{H}^{\infty}\left(\Omega_{\delta}\right)$ and the series $\sum_{n=0}^{\infty} \frac{f^{(n)}(\cdot)}{n !} z^{n}$ converges to $f(\cdot+z)$ in $\mathcal{H}^{\infty}\left(\Omega_{\delta}\right)$. Consequently, $U:=\Omega_{\delta} \cap \mathbb{R}$ satisfies $K \subset U$ and $\sum_{n=0}^{\infty} \frac{f^{(n)}(\cdot)}{n !} t^{n}$ converges to $f(\cdot+t)$ in $\mathcal{E}_{(\omega)}(U)$ for every $|t|<\delta$. From where it follows

$$
\langle\mu, \phi(t)\rangle=\sum_{n=0}^{\infty} \frac{\left\langle\mu, f^{(n)}\right\rangle}{n !} t^{n}
$$

for $|t|<\delta$. The same argument shows that $\mu \circ \phi(t)$ is real-analytic in a neighborhood of any point.

If $f$ is an entire function, we already know that $\phi$ is weakly analytic, and from the Cauchy inequalities it is easy to see that for every compact interval $I$ in $\mathbb{R}$ and every continuous seminorm $q$ in $\mathcal{E}_{(\omega)}(\mathbb{R})$ and each $m \in \mathbb{N}$ we have

$$
\sup _{t \in I} \sup _{j \in \mathbb{N}} \frac{q\left(\phi^{(j)}(t)\right)}{j ! m^{j}}<\infty
$$

and we use [7, Proposition 10] to conclude. In the Roumieu case, we have that $\mathcal{E}_{(\omega)}(\mathbb{R}) \hookrightarrow \mathcal{E}_{\{\omega\}}(\mathbb{R})$ continuously and continuous linear operators are topologically real analytic.

Proposition 4.9. Let $f \in \mathcal{A}(\mathbb{R})$ be given and assume that $\mathcal{E}_{*}(\mathbb{R})$ is closed by composition. Then, the map

$$
T_{f}: \mathcal{E}_{*}\left(\mathbb{R}^{N}\right) \rightarrow \mathcal{E}_{*}\left(\mathbb{R}^{N}\right), g \rightarrow f \circ g
$$

is real-analytic. If $f$ is an entire function, then $T_{f}$ is topologically real analytic. 
Proof. Put $E:=\mathcal{E}_{(*)}\left(\mathbb{R}^{N}\right)$ and fix $\alpha \in \mathcal{A}(\mathbb{R}, E)$. We have to show that $\beta:=T_{f} \circ \alpha \in \mathcal{A}(\mathbb{R}, E)$. We already proved that $\beta \in C^{\infty}(\mathbb{R}, E)$ and for every continuous seminorm $p$ in $E$ there is a continuous seminorm $q$ in $E$ such that

$$
p\left(\beta^{(n)}(t)\right) \leq \sum \frac{n !}{k_{1} ! \ldots k_{n} !} q\left(f^{(k)} \circ \alpha(t)\right)\left(\frac{q\left(\alpha^{\prime}(t)\right)}{1 !}\right)^{k_{1}} \ldots\left(\frac{q\left(\alpha^{(n)}(t)\right)}{n !}\right)^{k_{n}} .
$$

We denote $v_{m}(j):=\frac{1}{m^{j}}$ and we fix a compact interval $I \subset \mathbb{R}$. We apply $[7$, Proposition 10] to find $m \in \mathbb{N}$ (which depends on $q$ in the weakly analytic case and which does not depend on $q$ if $\alpha$ topologically real analytic) with the property

$$
\sup _{t \in I} \sup _{j \in \mathbb{N}} \frac{q\left(\alpha^{(j)}(t)\right)}{j !} v_{m}(j) \leq C<+\infty .
$$

Since $\{\alpha(t): t \in I\}$ is a bounded set in $E$, by Lemmas 4.1 or 4.3 there is a continuous seminorm $r$ on $E$ such that

$$
q\left(f^{(k)} \circ \alpha(t)\right) \leq r\left(f^{(k)}\right)
$$

for all $k \in \mathbb{N}$ and $t \in I$. We apply Lemma $4.8\left(\right.$ since $\left.\phi^{(n)}(0)=f^{(n)}\right)$ and $[7$, Proposition 10] to find $m$ large enough so that

$$
\sup _{k \in \mathbb{N}} \frac{r\left(f^{(k)}\right)}{k !} v_{m}(k) \leq D<+\infty .
$$

Here $m$ is independent of $r$ if $f$ is entire. Hence, for every $t \in I$, we have

$$
\begin{aligned}
p\left(\beta^{(n)}(t)\right) & \leq \sum \frac{n !}{k_{1} ! \ldots k_{n} !}\left(D m^{k} k !\right)(C m)^{k_{1}} \ldots\left(C m^{n}\right)^{k_{n}} \\
& =D \sum \frac{n !}{k_{1} ! \ldots k_{n} !} m^{k} k ! C^{k} m^{n} .
\end{aligned}
$$

Here we used that $k_{1}+\cdots+k_{n}=k$ and $k_{1}+\cdots+n k_{n}=n$. Consequently

$$
\begin{aligned}
\frac{p\left(\beta^{(n)}(t)\right)}{n !} v_{m}(n) & \leq D \sum \frac{n !}{k_{1} ! \ldots k_{n} !}(m C)^{k} \\
& \leq D(2 m C)^{n} .
\end{aligned}
$$

We finally take $\ell \in \mathbb{N}, \ell>2 m^{2} C$ and we obtain

$$
\sup _{t \in I} \sup _{n \in \mathbb{N}} \frac{p\left(\beta^{(n)}(t)\right)}{n !} v_{\ell}(n) \leq D,
$$

where $\ell$ depends on the seminorm if $\alpha \in \mathcal{A}(\mathbb{R}, E)$ and $f$ is real-analytic, whereas $\ell$ is independent on the seminorm in case $\alpha \in \mathcal{A}_{t}(\mathbb{R}, E)$ anf $f$ is an entire function. We apply again [7, Proposition 10] to finish the proof. 


\section{References}

[1] Appell, J. and Zabrejko, P. P., Nonlinear superposition operators, Cambridge: Cambridge University Press, 1990.

[2] Appell, J., Nazarov, V. I. and Zabrejko, P. P., Composing infinitely differentiable functions, Math. Z., 206 (1991), 659-670.

[3] Björck, G., Linear partial differential operators and generalized distributions, Ark. Mat., 6 (1965), 351-407.

[4] Bochnak, J., Analytic functions in Banach spaces, Studia Math., XXXV (1970), 273292.

[5] Boman, J., Differentiability of a function and of its compositions with functions of one variable, Math. Scand., 20 (1967), 249-268.

[6] Boman, J. and Hörmander, L., Classes of infinitely differentiable functions, mimeographed notes, Stockholm, 1962.

[7] Bonet, J. and Domanski, P., Real Analytic Curves in Fréchet Spaces and Their Duals, Monatsh. Math., 126 (1998), 13-36.

[8] Braun, R. W., Meise, R. and Taylor, B. A., Ultradifferentiable functions and Fourier Analysis, Results Math., 17 (1990), 206-237.

[9] Bruna, J., On inverse-closed algebras of infinitely differentiable functions, Studia Math., LXIX (1980), 59-68.

[10] Constantine, G. M., and Savits, T. H., A multivariate Faá di Bruno formula with applications, Trans. Amer. Math. Soc., 348 (1996), 503-520.

[11] Dyn'kin, E. M., Pseudoanalytic extension of smooth functions. The uniform scale, Amer. Math. Soc. Transl., 115 (2), (1980), 33-58.

[12] El Kinani, A. and Oudadess, M., Entire functions and m-convex structure in commutative Baire algebras, Bull. Belg. Math. Soc. Simon Stevin, 4 (1997), 685-687.

[13] Fernández, C., Galbis, A. and Jornet, D., w-hypoelliptic differential operators of constant strength, J. Math. Anal. Appl., 297 (2004), 561-576.

[14] Gómez-Collado, M. C., Ultradistribuciones casi-periódicas y operadores de convolución elípticos, Doctoral Thesis, Valencia, 2000.

[15] Jarchow, H., Locally convex spaces, B. G. Teubner Stuttgart, 1981.

[16] Komatsu, H., Ultradistributions. I. Structure theorems and a characterization, J. Fac. Sci. Univ. Tokyo Sect. IA Math., 20 (1973), 25-105.

[17] Krantz, S. and Parks, H., A primer on real analytic functions, Birkhäuser, Basel, 1992.

[18] Kriegl, A. and Michor, P. W., The convenient setting for global analysis, Math. Surveys Monogr., 53. American Mathematical Society, Providence, RI, 1997.

[19] Malliavin, P., Calcul symbolique et sous-algèbres de $L_{1}(G)$, Bull. Soc. Math. France, 87 (1959), 181-190.

[20] Mitiagin, B., Rolewicz, S. and Zelazko, W., Entire functions in B-algebras, Studia Math., XXL (1962), 291-306.

[21] Neelon, T., Ultradifferentiable functions on lines in $\mathbb{R}^{n}$, Proc. Amer. Math. Soc., 127 (1999), 7, 2099-2104.

[22] A correction to: "Ultradifferentiable functions on lines in $\mathbb{R}^{n}$ ", Proc. Amer. Math. Soc., 131 (2003), 991-992.

[23] Peetre, J., Concave majorants of positive functions, Acta Math. Acad. Sci. Hungaricae, 21 (1970), 327-333.

[24] Pérez, P. and Bonet, J., Barrelled locally convex spaces, North-Holland Mathematics Studies, 131. Notas de Matemática [Mathematical Notes], 113. North-Holland Publishing Co., Amsterdam, 1987.

[25] Petzsche, H. J. and Vogt, D., Almost Analytic Extension of Ultradifferentiable Functions and the Boundary Values of Holomorphic Functions, Math. Ann., 267 (1984), 17-35.

[26] Roumieu, Ch., Ultra-distributions définies sur $\mathbb{R}^{n}$ et sur certaines classes de variétés différentiables, J. Anal. Math., 10 (1962/1963), 153-192.

[27] Rudin, W., Division in algebras of infinitely differentiable functions, J. Math. Mech., 11 (1962), 797-809. 
[28] Siciak, J., A characterization of analytic functions of $n$ real variables, Studia Math., XXXV (1970), 293-297.

[29] Siddiqi, J. A., and Ider, M., Characterization of inverse-closed Beurling classes, Math. Proc. Cambridge Philos. Soc., 105 (1989), 495-501.

[30] Timan, A. F., Theory of approximation of functions of a real variable, Fitzmatgiz, Moscow, 1960; English transl., Macmillan, New York, 1963.

[31] Zelazko, W., A non-m-convex algebra on which operate all entire functions, Ann. Polon. Math., 46 (1985), 389-394. 